\title{
UNIVERSIDADE FEDERAL DO RIO GRANDE DO SUL
}

CINTED - Centro Interdisciplinar de Novas Tecnologias na Educação

Ciclode Palestras: Inovações em Tecnologia na Educação: Processos e Produtos

\section{RELATO DA CONSTRUÇÃO DO COLISÕES: PROBLEMATIZAÇÃO DA INTERFACE}

Gabriela Trindade Perry - (UFRGS) - trindade@producao.ufrgs.br Agostinho Serrano Andrade Neto - (ULBRA) - serrano@ulbra.tche.br Fernando Gonçalves Amaral - (UFRGS) - amaral@producao.ufrgs.br

Porto Alegre, Setembro de 2004 
Relato da construção do Colisões: problematização da interface

\author{
Gabriela Trindade Perry* \\ Agostinho Serrano Andrade Neto \\ Fernando Gonçalves Amaral ${ }^{* * *}$
}

\title{
Resumo
}

O objetivo deste trabalho é fazer um relato do desenvolvimento do software Colisões, para o ensino de Equilíbrio Químico e Princípio de Le Chatelier, no nível secundário, a partir da exposição de problemas que surgiram durante a construção de sua interface gráfica. Argumenta-se que, como muitas das decisões fundamentais tomadas durante o desenho da interface são altamente dependentes do contexto de trabalho, as guidelines, os checklists, as heurísticas e os testes de usabilidade não são suficientes para fundamentar tais decisões. Ressalta-se também que as necessidades dos usuários de softwares educacionais são muito diferentes daquelas de usuários de softwares convencionais. Torna-se necessário; portanto, o emprego de um método que sistematize o ciclo de desenvolvimento, e que promova a integração do conhecimento e das experiências dos diversos atores do envolvidos no processo.

Palavras - chave: interação homem - computador; desenvolvimento de software educacional; ciclo de vida de software educacional.

\begin{abstract}
The aim of this work is to report the development of the software "Colisöes", constructed to the teaching of chemical equilibrium concepts and the principle of Le Chatelier, wich is supposed to be used both by pre-college or undergraduating students, through the exposicion of the problems that arised during the construction of the graphical interface of the software. It is argued that, as many of the decisions taken during the construction of the software are highly context dependent, the guidelines, checklists, the heuristics and the usability tests, commonly used in designing a software, do not provide enough support for all the decision-making process involved in the construction process itself. Is is also stressed that the needs of the users of educational softwares may be quite different of the conventional software user. It is necessary then the employment of a method that make it sistematic the development cycle, and that promotes the integration of the experiences and knowledge of the different actors involved in the process.
\end{abstract}

Keywords: Human - computer interaction; educacional software development; educacional software life cicle.

\section{Introdução}

Para projetar softwares educacionais eficientes, é preciso compreender o grau de dificuldade de produzí-los. Pode-se pensar sobre a questão a partir de três pontos de vista. O primeiro, a respeito das abordagens teóricas disponíveis, coloca-nos dois problemas: nenhuma delas pode ser diretamente transporta para o universo dos softwares (CAMPOS et al., 1996), e segundo, a tecnologia disponível hoje não permite "emular" o conhecimento do professor, prejudicando a avaliação do aluno, fazendo com que softwares que se proponham tal empresa corram o riso de serem enquadrados como comportamentalistas. O segundo ponto de vista é o daqueles que relatam problemas durante a inserção, tais como Kaplan (1994), citada por Campos et al. (1996); Machado (1994) e Grünberg (1994). O terceiro ponto de vista é relativo à própria natureza da tarefa de desenvolvimento,

\footnotetext{
* Estudante de Mestrado Acadêmico - Bacharel em Design - Programa de Pós Graduação em Engenharia de Produção - PPGEP/UFRGS - trindade@producao.ufrgs.br - http://www.producao.ufrgs.br

"Professor adjunto - Doutor em Física - Departamento de Matemática - serrano@ulbra.tche.br - http://www.ulbra.br

"'Professor adjunto - Doutor em Sciences de la Santé Publique, orientação Ergonomia - Programa de Pós Graduação em Engenharia de Produção - PPGEP/UFRGS - amaral@producao.ufrgs.br - http://www.producao.ufrgs.br 
essencialmente interdisciplinar. Por exemplo, Gomes (2003) refere-se ao fato de que no processo de concepção de software educacional há uma diferença significativa entre as representações que designers, programadores e professores têm acerca dos processos de ensino / aprendizagem. Além disso, cita que mesmo as soluções geradas em centros de pesquisa tendem a abordar o problema do desenvolvimento de aplicações educativas de forma não sistemática.

Wegerif (2004) propõe a construção de interfaces que estimulam trocas educacionais do tipo IDRF: Iniciation, Discussion, Reply, Feedback, sustentando que interfaces deste tipo aumentaram o aprendizado entre crianças de nove a dez anos, nos softwares em que tal modelo foi aplicado, abordando tópicos em ensino de ciências e matemática, inglês e cidadania (em colégios primários no Reino Unido). Stern (2000) apresenta um processo de construção, baseado na observação do ambiente de sala de aula e em pesquisas prévias, que resultaram nos seguintes em uma série de itens de design. No entanto, para Campos et al. (1996), a proposição de guidelines (ou princípios de design) não é suficiente para tratar da complexidade inerente à construção de softwares educionais. Os usuários para os quais tais ferramentas são construídas têm necessidades especiais, que não são contempladas nem pelas metodologias de desenvolvimento de Engenharia de Software, nem pelas de Ergonomia.

\subsection{Uma abordagem participativa para o desenvolvimento de interfaces: Design Centralizado no Usuário}

Uma alternativa é empregar, durante o desenvolvimento, a metodologia do Design Centrado no Usuário (UCD), que, segundo Gould (1995), se apóia em: métodos de análise da tarefa e dos usuários; testes de protótipos com usuários reais e em design iterativo. Cybis (2003) cita que o UCD traz resultados positivos porque não considera o projeto da interface com o usuário com base no trabalho prescrito, ou seja: privilegia o desempenho do usuário em sua tarefa, concebendo sistemas adaptados às suas características e a seus objetivos.

O UCD não é uma metodologia formalmente expressa, podendo ser melhor interpretada como uma "filosofia de design". Existem, contudo, métodos bem estruturados para contemplar os itens de UCD. A análise da tarefa, é, por exemplo, um assunto-chave para a Ergonomia. Destaca-se a AET (Análise Ergonômica do Trabalho), preconizadas por Wisner (1994). O trabalho desenvolvido pelo STICEF - Sciences et Technologies de l'Information et de la Communication pour l'Éducation et la Formation - (STICEF, 2004) é um exemplo da proposta de desenvolvimento de software educacional, a partir de métodos de análise da tarefa.

O estudo dos testes com o usuário, também conhecido como design walkthrough (WARLTON et al., 1992), por sua vez, é um assunto de grande importância para o estudo da interação homem - computador; seu uso não se restringe àqueles que praticam UCD. Rubin (1994), por sua vez, detalha diversos tipos de testes, em relação à preparação, condução, avaliação, interpretação e momento de aplicá-los.

O último ponto da metodologia UCD é o design iterativo. De acordo com a definição de Myers (1994), durante o processo de design iterativo, "a interface é prototipada e redesenhada repetidamente, e testada em usuários reais". A prática de design iterativo apresenta; porém, diversas dificuldades. Bailey (1993) afirma que dados experimentais dão suporte à idéia de que mudanças introduzidas para resolver um problema de usabilidade acabam gerando outro problema. Outra informação interessante extraída deste artigo é que 
a primeira iteração dos designers apresentou menos "erros" de design do que a última dos programadores.

Uma abordagem para o desenvolvimento de interfaces educacionais, com origens no UCD é LCD (Learner Centered Design), comprometida, de acordo com Solloway (1996), com o construtivismo de Piaget e Vygotsky e com o construcionismo de Papert. Quintana et al. (2000), Solloway (1994), Squires (1996) e Squires (1999) são algumas referências a LCD.

\section{Colisões: histórico e propostas}

A proposta do Colisões foi, desde o início, deixar evidente a natureza dinâmica do equilíbrio químico, especialmente em nível microscópico. No ensino de Química, são propostos 3 níveis de representação: macroscópico, microscópico e simbólico. Estudos empíricos mostram que a compreensão dos níveis microscópico e simbólico é especialmente difícil para os estudantes, por serem representações de algo invisível e / ou abstrato, enquanto o raciocínio deles se baseia fortemente em informações sensoriais (WU et al., 2001). O software propõe-se; portanto, articular os três níveis de representação. No plano do desenvolvimento da interface gráfica; contudo, houveram alguns problemas.

\subsection{Problematização da interface do Colisões}

O Colisões é dividido em duas áreas. Na primeira, composta por uma tela, há quatro objetos de interação, que permitem configurar a temperatura do sistema e a concentração molar inicial dos reagentes (H2 e I2) e dos produtos (2HI). Na segunda área estão: a simulação em nível microscópico, os gráficos de velocidade da reação e de concentração de produtos e reagentes, um balão que muda de cor de acordo com o estágio em que se encontra a reação, e a unidade de Le Chatelier, onde os parâmetros concentração, pressão e temperatura podem ser alterados. Todos estes elementos da interface estão articulados, ou seja, a interferência em qualquer um deles modifica os demais.

Para facilitar a leitura deste artigo, classificou-se a interface em três categorias: entrada de dados (objetos de interação); saída de dados e modelagem da simulação. De uma maneira geral, as questões que envolvem a entrada de dados referem-se à escolhas do tipo: os controles devem se assemelhar aos equipamentos de um laboratório ou não? A figura 1 ilustra as duas possibilidades.
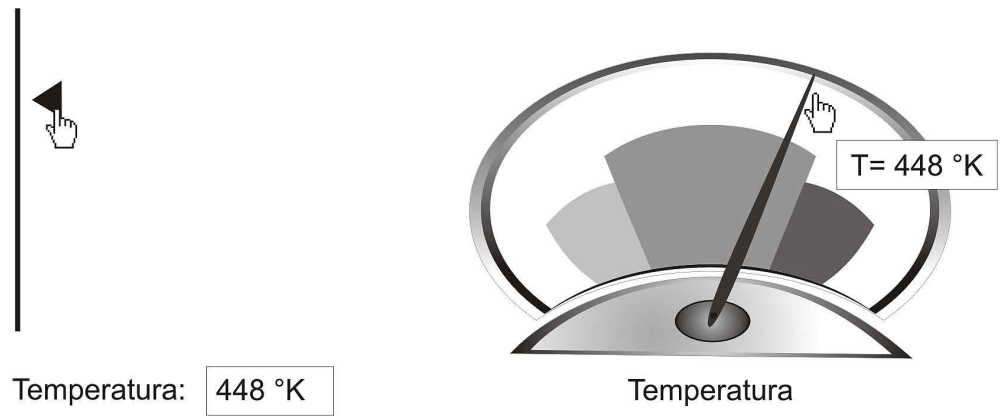

Figura 1 - objetos de interação 
O balão da reação é um exemplo de como o cuidado com a representação dos elementos de saída é fundamental. Na primeira vez que foi inserido, não era possível perceber que a reação era gasosa, pois o balão estava "aberto". Nesta reação, modificações no fator pressão não alteram o estado do sistema. A figura 2 mostra os dois desenhos do balão.
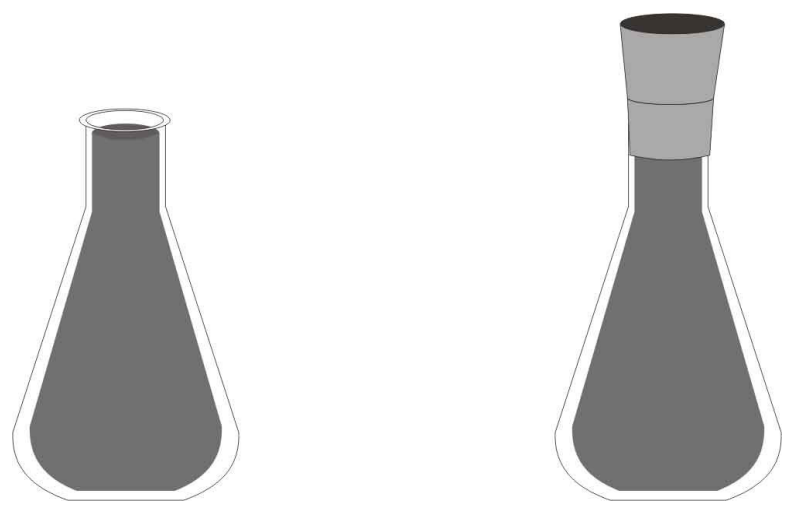

Figura 2 - balões sem rolha e com rolha

Em relação à aparência dos elementos de saída de dados, pode-se tomar o exemplo dos gráficos. À medida que são feitas mudanças no sistema, os estudante deve poder comparar os gráficos com as configurações que lhes deram origem. Coloca-se então a pergunta: como dar acesso a diversos gráficos, mostrando suas configurações iniciais e os estados finais de cada sistema? A figura 3 ilustra uma possibilidade.

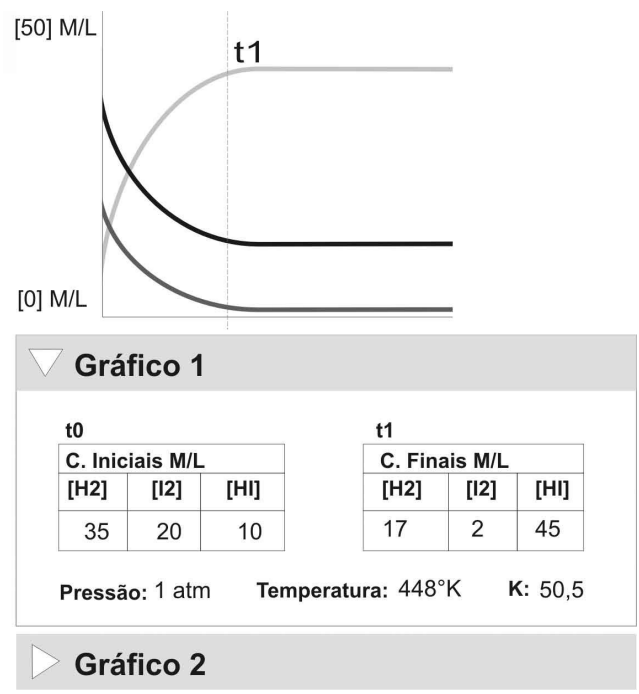

Figura 3 - estados iniciais e finais de dois sistemas

O último ponto abordado é refere-se ao modelo da simulação microscópica. Neste momento, deve ser determinada o quão próximo da realidade ele se posiciona. O que levar em consideração para determinar se uma colisão será efetiva? A orientação das moléculas 
será considerada? Quantos eixos serão visualizados? A rotação das moléculas será em quantos eixos? As reações intermediárias serão representadas? Todas as moléculas terão velocidades iguais? Estes foram algumas das perguntas que tiveram que ser respondidas antes de começar a construir a unidade de simulação. Seu modelo pode ser brevemente descrito da seguinte forma:

1. Um cálculo de probabilidade determina se a colisão será efetiva ou não (quanto maior a temperatura, maior a probabilidade de efetivar a colisão);

2. A orientação não é considerada, e as moléculas giram em torno de um eixo apenas $(\mathrm{z})$;

3. Apenas uma das etapas da reação é considerada.

4. Às moléculas são atribuídas velocidades diferentes, dentro de uma faixa que varia conforme a temperatura.

Do ponto de vista operacional; no entanto, a maior dificuldade estava em um aspecto que não foi percebido: como seriam definidas as posições iniciais das moléculas? Como a proposta é ser o mais fiel possível ao modelo científico, as moléculas tiveram suas posições iniciais $(\mathrm{x}, \mathrm{y})$ randomizadas dentro de uma área pré-determinada. Esta solução gerava um "bug" cada vez que duas moléculas ficavam sobrepostas: elas ficavam "grudadas" uma na outra. A causa deste problema; no entanto, não será discutida neste trabalho, pois não faz parte do escopo desta discussão. Posto este problema, a solução adotada foi a seguinte: ao posicionar uma molécula, sorteia-se uma coordenada, que depois é tirada do conjunto de coordenadas possíveis. Estas coodernadas estão "fora" da área da simulação, de modo que as moléculas vão gradualmente entrando na área. Uma vez que a molécula está dentro na área da simulação, não é possível sair. Esta solução será estendida à unidade Le Chatelier, de modo que, quando o volume diminui, algumas moléculas saem, e quanto aumentar, outras entram. A figura 4 traz um esquema da solução adotada.

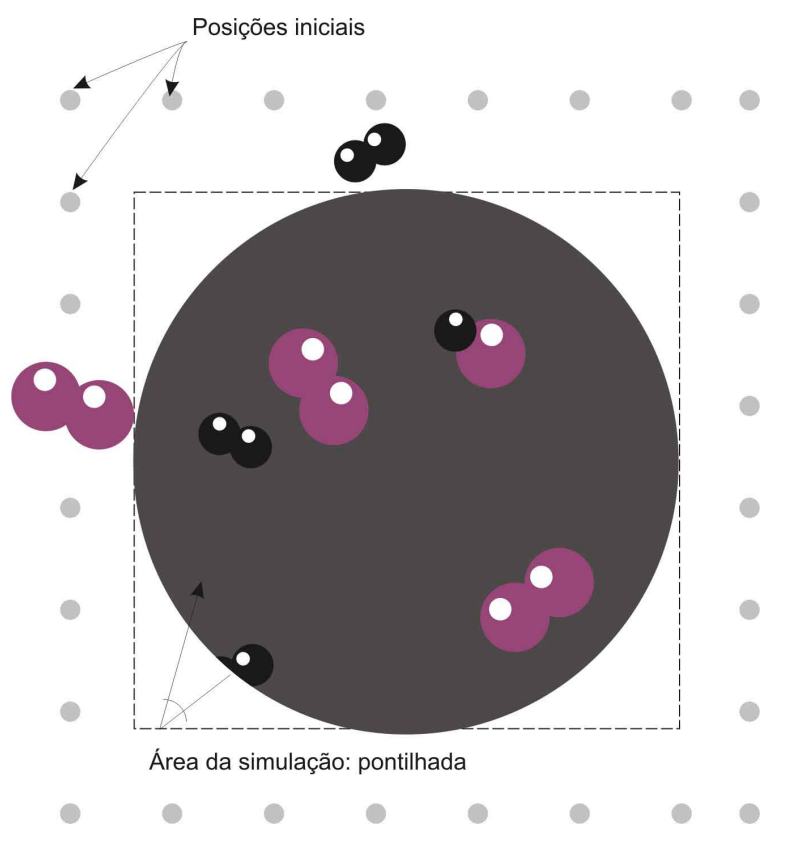

Figura 4 - modelo da simulação

\section{Conclusões}


Construir a interface de uma simulação para o ensino de ciências é certamente um grande desafio. É necessário fazer com que todos os membros da equipe se comuniquem, e que as habilidades de cada um possam ser aproveitadas ao máximo. Com este propósito, o Colisões passará a sua segunda versão, sendo construído através do M.A.R.S., um ciclo de desenvolvimento proposto por Pernin (1996). MARS propõe três espaços de trabalho dedicados respectivamente ao modelo da simulação, às associações e à representação, além de um quarto espaço de trabalho dedicado à integração dos anteriores. Em cada um destes espaços de trabalho participam atores pré-determinados, com tarefas bem delimitadas, considerando suas habilidades. Este processo leva à atividades de projeto paralelas, cada uma delas seguindo um ciclo de prototipagem individual. Estes ciclos de prototipagem são compostos pelas fases de especificações formais, geração do protótipo e avaliação. Segundo o autor, trata-se da "arquitetura de um ambiente integrado e adaptativo, que possibilita oferecer diferentes níveis de acesso, de acordo com as capacidades do aluno, o domínio estudado, o contexto de produção etc.".

\section{Referências Bibliográficas}

BAILEY, Greg. Iterative Methodology and Designer Training in Human Computer Interface Design. In: Conference on Human Factors in Computing Systems. 1993, Amsterdam. Anais... p. 198-205.

CAMPOS , Fernanda C.; ROCHA, Ana Regina C. Design Instrucional E Construtivismo: Em Busca De Modelos Para O Desenvolvimento De Software. In: Congresso Iberoamericano de Informática Educativa - RIBIE, 3. Santiago: 1996. Disponível em: < http://www.c5.cl/ieinvestiga/i_actas.htm > Acesso em: 05 jul. 2004.

CYBIS, Walter de Abreu. Engenharia de Usabilidade: Uma Visão Ergonômica. Florianópolis: s.ed. 2003.

GOMES, Alex Sandro; WANDERLEY, Eduardo Garcia. Elicitando Requisitos em Projetos de Software Educativo. In: WIE 2003 WORKSHOP BRASILEIRO DE INFORMÁTICA EDUCATIVA, 2003, Convergências Tecnológicas - Redesenhando as Fronteiras da Ciência e da Educação: Anais. Campinas: SBC, 2003. v. v.;

GOULD. John. D; LEWIS, Clayton. Designing for Usability: Key Principles and What Designers Think. Communications of the ACM. V. 28, n. 3. p. 300-312, mar. 1995.

GRÜNBERG, Jorge A. Profesores Y Computadores: Una Investigacion Sobre Los Factores Que Afectan El Uso De Computadores En Colegios Secundarios. In: Congresso Iberoamericano de Informática Educativa - RIBIE, 1. Santiago: 1994. Disponível em: < http://www.c5.cl/ieinvestiga/i_actas.htm > Acesso em: 05 ago. 2004.

MACHADO, Maria José. Atitudes Dos Professores Do $\mathbf{1}^{\mathbf{0}} \mathbf{E} \mathbf{2}^{\circ}$ Ciclos Do Ensino Básico Face Às Tecnologias $\mathrm{E}$ Sua Utilização Na Sala De Aula. In: Congresso Iberoamericano de Informática Educativa - RIBIE, 1. Santiago: 1994. Disponível em: < http://www.c5.cl/ieinvestiga/i_actas.htm > Acesso em: 05 ago. 2004.

MYERS, Brad. A. Challenges of HCI Design and Implementation. Interactions. Jan. 1994.

PERNIN, Jean-Philippe. M.A.R.S.. Un Modèle Opérationnel de Conception de Simulations Pédagogiques. Genoble : Université Joseph Fourier, 1996. Tese, Laboratoire CLIPS - IMAG, Université Joseph Fourier, 1996. 
QUINTANA, C; SOLLOWAY, Elliot; KRAJCIK, J. Exploring a Structured Definition of Learner Centered Design. In: International Conferences of Learning Sciences, 4. 2000, Mahwah: Erlbaum. Anais... p. 256-263.

RUBIN, Jeffrey. Handbook of usability testing: how to plan, design, and conduct effective tests. New York : John Wiley, 1994.

SOLLOWAY, Elliot. et al. Learner Centered Design: The Challenge for HCI in the 21th Century. Interactions. Abril. 1994.

Learning Theory in Practice: Case Studies of Learner Centered Design. In:

Conference on Human Factors in Computing Systems. 1996, Vancouver. Disponível em: < http://www.acm.org/sigchi/chi96/proceedings/papers/Soloway/es_txt.htm

>. Acesso em: 20 ago. 2004.

SQUIRES, David, PREECE, Jenny. Predicting Quality in Educational Software. Evaluating for Learning, Usability and the Synergy Between them. Interacting With Computers. V. 11, p. 467-483. 1999.

Usabilty and Learning: Evaluating the Potential of Educational Software. Computers and Education, v. 17, n.1, p. 15-22. 1996.

STERN, Judith. The Design of Learning Software: Principles Learned from the Computer as Learning Partner Project. Journal of Science Education and Technology. V. 9, n. 1. 2000 .

STICEF: Sciences et Technologies de l'Information et de la Communication pour

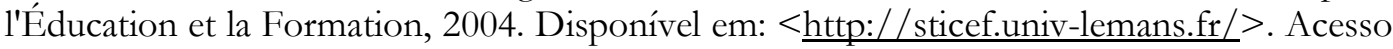
em: 01 de set. 2004.

WARLTON, Cathleen. et al. Applying Cognitive Walkthroughs to More Complex User Interfaces: Experiences, Issues and Recommendations. In: Conference on Human Factors in Computing Systems. 1992, Monterey. Anais... p. 331-388.

WEGERIF, Rupert. The Role of Educational Software as a Support for Teaching and Learning Conversations. Computers and Education (in press). 2004.

WISNER, Alain. Por dentro do trabalho: ergonomia, método e técnica. Traduzido por Roberto Leal Ferreira. São Paulo: UNESP, [1994].

Wu, H., K.; Krajcik, S., J.; Solloway, E. (2001). Promoting Understanding of Chemical Repesentations: Students Use of a Visualization Tool in the Classroom. Journal of Research in Science Teaching, Vol. 38, Abril, 821-842. 